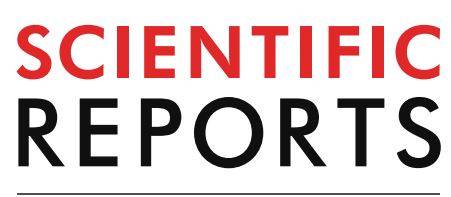

natureresearch

Check for updates

\title{
OPEN Kinome profiling of peripheral blood mononuclear cells collected prior to vaccination reveals biomarkers and potential mechanisms of vaccine unresponsiveness in pigs
}

\author{
Sean W. L. Lipsitt ${ }^{1,2}$, James Wilkinson ${ }^{3,6}$, Erin Scruten ${ }^{1}$, Antonio Facciuolo ${ }^{1}$, \\ Connor Denomy ${ }^{1,2,5}$, Philip J. Griebel ${ }^{1,4}$, Anthony Kusalik ${ }^{5}$, Graham Plastow ${ }^{3}$ \& \\ Scott Napper ${ }^{1,2 \bowtie}$
}

Inter-individual variance in host immune responses following vaccination can result in failure to develop protective immunity leaving individuals at risk for infection in addition to compromising herd immunity. While developing more efficacious vaccines is one strategy to mitigate this problem, predicting vaccine responsiveness prior to vaccination could inform which individuals require adjunct disease management strategies. To identify biomarkers of vaccine responsiveness, a cohort of pigs $(n=120)$ were vaccinated and pigs representing the high $(n=6 ; 90$ th percentile) and low ( $n=6 ; 10$ th percentile) responders based on vaccine-specific antibody responses following vaccination were further analyzed. Kinase-mediated phosphorylation events within peripheral blood mononuclear cells collected prior to vaccination identified 53 differentially phosphorylated peptides when comparing low responders with high responders. Functional enrichment analysis revealed pro-inflammatory cytokine signaling pathways as dysregulated, and this was further substantiated by detection of higher $(p<0.01)$ concentrations of interferon-gamma in plasma of low responders compared to high responders prior to vaccination. In addition, low responder pigs with high plasma interferon-gamma showed lower $(p<0.01)$ birth weights than high responder pigs. These associations between vaccine responsiveness, cytokine signaling within peripheral immune cells, and body weight in pigs provide both evidence and insight into potential biomarkers for identifying low responders to vaccination.

Infectious diseases currently represent the largest source of economic losses to the livestock industry ${ }^{1}$. With increased regulation against antibiotic usage in livestock, there is an even greater need to manage infectious diseases through alternative strategies like vaccination ${ }^{2}$. An inherent challenge with vaccination is the quantitative and qualitative variability in immune responses. Individuals that fail to generate an immune response to vaccination remain at risk for infection and compromise the protection afforded through herd immunity ${ }^{3}$. Unfortunately, the empirical approach that is typically taken for vaccine development does little to enhance our understanding of the mechanisms or molecular events promoting differential immune responses to vaccination. Biomarkers predictive of vaccine responsiveness could enable more strategic management of both vaccination programs and

\footnotetext{
${ }^{1}$ Vaccine and Infectious Disease Organization-International Vaccine Centre (VIDO-InterVac), University of Saskatchewan, Saskatoon, SK, Canada. ${ }^{2}$ Department of Biochemistry, Microbiology and Immunology, University of Saskatchewan, Saskatoon, SK, Canada. ${ }^{3}$ Department of Agricultural, Food and Nutritional Science, University of Alberta, Edmonton, AB, Canada. ${ }^{4}$ School of Public Health, University of Saskatchewan, Saskatoon, SK, Canada. ${ }^{5}$ Department of Computer Science, University of Saskatchewan, Saskatoon, SK, Canada. 6 Illumina Centre, Great Abington, Cambridge, UK. ${ }^{\varpi}$ email: scott.napper@usask.ca
} 
animal health. Furthermore, understanding the molecular events associated with vaccine responsiveness could guide the development of more effective vaccines.

Within human and livestock populations, biomarkers capable of predicting vaccine immunogenicity and efficacy could be used to reduce the costs associated with vaccine production and testing ${ }^{4}$. This need has spawned 'systems vaccinology' approaches that define the host factors contributing to immune responses to a variety of vaccines $^{5-9}$. As vaccine-induced immune responses have shown significant heritability in animals ${ }^{10}$, identifying traits indicative of immune responsiveness could likely be applied for marker-assisted selection of animals ${ }^{11}$. In humans, a genome-wide association study attributed $\sim 30 \%$ of the individual variability in the humoral immune response to measles vaccine to polymorphisms in immune response genes and human leukocyte antigen alleles ${ }^{12}$. Other genome-wide assessments of vaccine-induced immune responses in humans have identified associations with polymorphisms in antigen presentation proteins and lymphocyte receptors such as toll-like receptors ${ }^{13}$, signaling lymphocyte activation molecule ${ }^{14}$, cytokines, and cytokine receptors ${ }^{15,16}$.

While genetic variability can influence individual immune responsiveness to vaccination, the situation is further complicated by dynamic variables including nutritional, situational, environmental, and an individual's health status. There are populations in which vaccines have proven to be less effective, specifically the elderly ${ }^{17}$, the young ${ }^{18}$, the obese $\mathrm{e}^{19,20}$, and individuals with health factors that influence immune function, including stress ${ }^{21}$, autoimmunity ${ }^{22,23}$, and infection ${ }^{24}$. As such, an individual's response to vaccination may be compromised regardless of their genetic predispositions. This necessitates the identification of more dynamic correlates of vaccine responsiveness. Dynamic variables affecting vaccine-induced immune responses have prompted immune-profiling efforts through transcriptional analysis, which have begun to illustrate transcriptional signatures associated with responses to various vaccines. For example, transcriptomic analysis of whole blood combined with polychromatic flow cytometry has identified both gene expression markers and immune cell-types characterizing vaccine-induced immune response processes to yellow fever-17D vaccination ${ }^{8,25}$. Similarly, transcriptional analysis of peripheral blood mononuclear cells (PBMCs) has revealed differential gene expression as correlates of hemagglutination inhibition titer responses at early time-points following influenza vaccination ${ }^{5}$. While transcriptional and cellular events have been explored in the hours following vaccination of livestock animals ${ }^{26}$, biomarkers that are predictive of the vaccine-induced immune responses prior to vaccination remain elusive, and the mechanisms that underlie vaccine responsiveness have yet to be fully defined.

Characterizing global cellular kinase activity (kinome analysis) is emerging as a powerful approach for understanding complex biology. Dynamic kinases catalyze phosphorylation events that regulate numerous cellular processes and often initiate a rapid functional change within cells. Species-specific peptide arrays used for high throughput kinome characterizations have delineated the complex biology associated with immune competence ${ }^{27}$ and biomarker discovery ${ }^{28}$. Within economically important livestock species, kinome analysis has revealed differential phosphorylation of host signaling pathways in response to mycotoxin consumption in pigs ${ }^{29}$ and Mycobacterium avium subsp. paratuberculosis infection in cattle ${ }^{30,31}$. As such, the genetic and dynamic traits influencing vaccine responsiveness may be better understood by characterizing kinase activity within immune cells at the time of vaccination.

Previously, transcriptional analysis was conducted on PBMCs collected from the pigs used in this study prior to vaccination ${ }^{32}$. Comparative analysis between the high and low responders prior to vaccination could only detect statistically significant differences in gene expression after vaccination. Given the ability of kinome analysis to investigate mechanisms of host phenotypes, kinome analysis was used to analyze cell signaling events within PBMCs collected from pigs immediately prior to vaccination. Pigs were subsequently stratified on the basis of serum Mycoplasma hyopneumoniae (M. hyopneumoniae)-specific IgG titer induced by RespiSure-One vaccination and grouped as high (HR) and low (LR) responders. While specific T cell responses can be used as a metric for vaccine responses, the current study uses serum IgG titers as the metric for vaccine responsiveness. Kinome analysis of PBMC lysates revealed different phosphorylation patterns when comparing cohorts of HR and LR pigs, where phosphorylation differences implicated pro-inflammatory cytokine signaling. Subsequent analysis of plasma cytokine concentrations confirmed higher concentrations of plasma interferon-gamma (IFN $\gamma$ ) in LR pigs compared to HR pigs at the time of vaccination. Information obtained from kinome analysis was then used to improve the understanding of host factors influencing vaccine responsiveness and to search for a specific biomarker that was predictive of vaccine responsiveness at the time a vaccine is administered.

\section{Results}

Differential antibody responses to vaccination. Pigs were vaccinated with an M. hyopneumoniae vaccine (Respisure-One) and serum IgG antibody-responses were quantified by IDEXX ELISA at 11 days after booster vaccination. There was a broad variation in serum $M$. hyopneumoniae-specific IgG titer responses to the vaccine, with $\log _{2}$ titer values ranging from 5.85 to 13.67. Based on titers and sample availability, six pigs above the 90th percentile and six pigs below the 10th percentile of serum M. hyopneumoniae-specific IgG titer were selected as high (HR) and low (LR) responders, respectively (Fig. 1a). The HR and LR pigs differed $(\mathrm{p}<0.0001)$ in serum M. hyopneumoniae-specific IgG titer (Fig. 1b) and were selected for subsequent analyses to determine biomarkers of vaccine responsiveness.

Kinome analysis of high and low responders. Kinome analysis was performed on PBMCs isolated immediately prior to primary vaccination and restricted to animals in the HR and LR cohorts. Of the 282 unique peptide targets represented on the arrays, 161 were consistently $\left(\chi^{2}>0.01\right)$ phosphorylated among the nine technical replicates. Therefore, characterization of the HR and LR kinome profiles was restricted to these 161 consistently phosphorylated peptides. t-distributed Stochastic Neighbour Embedding (t-SNE) analysis of HR and LR datasets was used to cluster similar kinome profiles of pigs. $t-S N E$ of the kinome data grouped HR and 

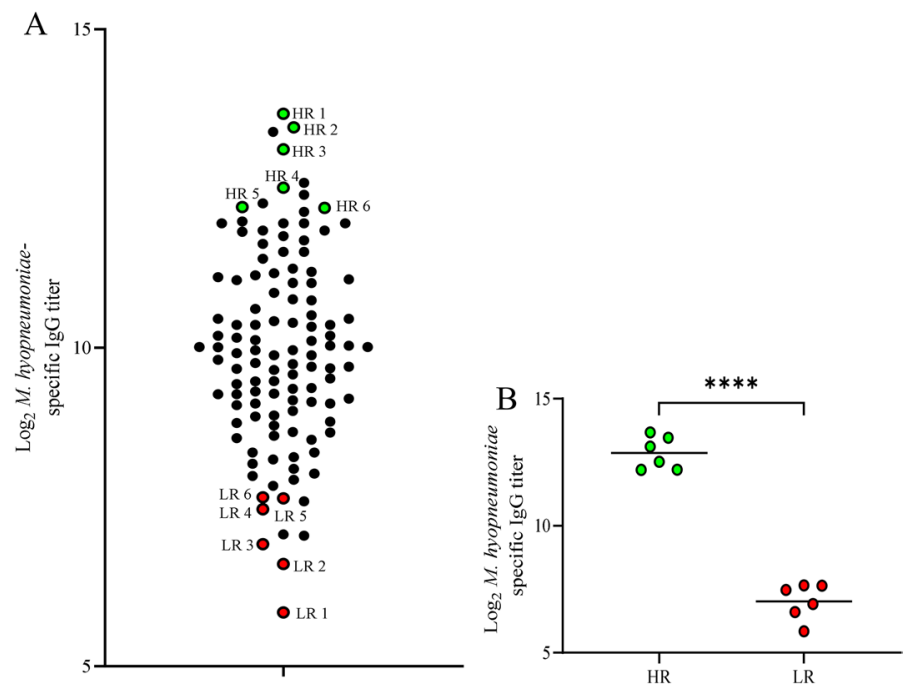

Figure 1. Variability in vaccine responsiveness in a population of pigs vaccinated with RespiSure-One and selecting high and low responders. (A) Serum M. hyopneumoniae-specific IgG titer in vaccinated pigs. High responders (HR; green) and low responders (LR; red) were selected from the cohort. (B) Serum $M$. hyopneumoniae-specific IgG titer of $\mathrm{HR}$ and LR pigs 35 days following primary vaccination; lines represent the mean. $\mathrm{n}=117$ in (A) and $\mathrm{n}=6$ for both HR and LR groups in $(\mathbf{B})$; $^{* * *} \mathrm{p}<0.0001$, unpaired two-tailed Student's t-test.

A

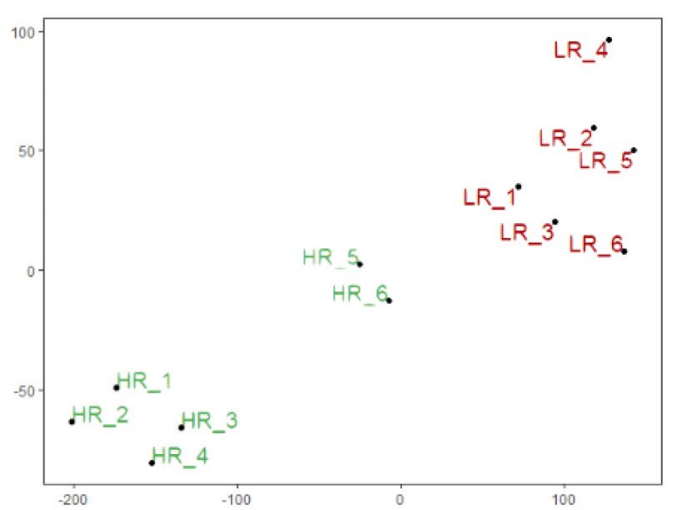

B
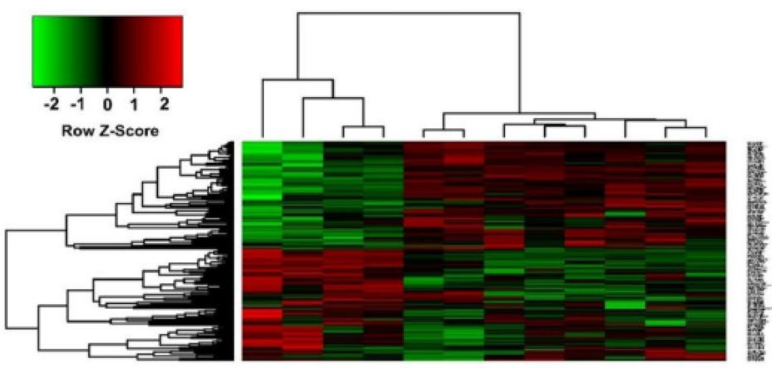

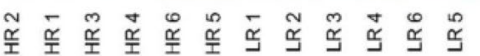

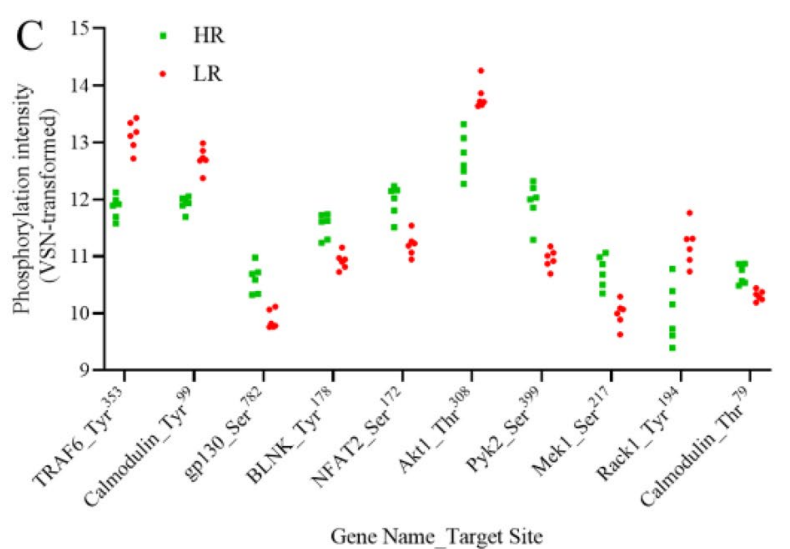

Figure 2. Differential phosphorylation patterns within PBMCs collected from high and low responder pigs prior to vaccination. (A) t-Distributed Stochastic Neighbor Embedding analysis and (B) Hierarchical clustering of high (HR) and low (LR) responders based on differential phosphorylation patterns in PBMCs collected prior to vaccination. (C) Top 10 most differentially phosphorylated peptides between HR (green) and LR (red) pigs prior to vaccination. $\mathrm{n}=6$ for both HR and LR groups. 
LR pigs based on their serum IgG titer following booster vaccination (Fig. 2a). Hierarchical clustering based on differential phosphorylation of peptides prior to primary vaccination showed a clear clustering of pigs reflecting their vaccine responsiveness 11 days after booster vaccination (Fig. 2b). In both t-SNE and hierarchical clustering analyses, HR 5 and HR 6 show distinct clustering separate from HR 1-4 and LR 1-6 which may suggest unique signaling events within these PBMCs prior to vaccination.

Consistent with the observation that HR and LR pigs have unique phosphorylation patterns prior to vaccination, 53 individual peptides were found to be differentially phosphorylated $(\mathrm{p}<0.05)$ when comparing HR and LR pigs (Table 1). Of the peptides represented on the array, the peptides which showed the greatest difference on the basis of statistical value included innate immunity-related genes and cell signaling mediators (Fig. 2c). Individual peptides differentially phosphorylated when comparing HR and LR pigs were used for pathway analysis to identify cell signaling pathways in which the peptides may be involved.

Pathway over-representation analysis. To further analyze the variations in signaling within the PBMCs of HR and LR pigs collected prior to vaccination, the 53 differentially phosphorylated peptides were subjected to over-representation analysis (ORA) using InnateDB. Of the 53 peptides queried, InnateDB designated 27 (51\%) to belong to Innate Immune System (Table 2). Within the pathway analysis dataset, there was a high representation of cytokine signaling entities, such as TNFalpha, $I L-7$ signaling, $I L-2, I L-3$, and IL-6, in addition to innate immunity signaling pathways including RANKL signaling and JAK-STAT pathway and regulation (Table 2). Altogether, ORA of the differentially phosphorylated genes between HR and LR suggests differential signaling of pro-inflammatory cytokines and innate immune signaling in the PBMCs of HR and LR pigs prior to vaccination.

Cytokine profiling of high and low responders. To further explore the observation that pro-inflammatory cytokine signaling of HR and LR pigs was differentially regulated, nine cytokines (IFNa, IFN $\gamma$, IL- $1 \beta$, IL- 6 , IL-8, IL-12, IL-13, IL-17 $\alpha$ and TNF $\alpha$ ) were assayed in plasma collected prior to primary vaccination of HR and LR pigs. LR pigs had higher $(\mathrm{p}=0.0087)$ plasma concentration of IFN $\gamma$ compared to HR pigs (Fig. 3a). A possible difference in TNFa $(p=0.18)$ (Fig. 3b) and IL-1 $(p=0.061)$ (Fig. 3c) plasma concentrations was also observed when comparing LR and HR pigs. HR and LR pigs showed no significant difference ( $p>0.05)$ in IFNa, IL-6, IL-8, IL-12, IL-13, or IL-17 a plasma concentrations prior to primary vaccination. Finally, within the HR and LR pigs, plasma IFN $\gamma$ concentration prior to primary vaccination was negatively correlated $(\mathrm{r}=-0.68, \mathrm{p}<0.05)$ with serum M. hyopneumoniae-specific IgG titer (Fig. 3d). Correlation analyses were not conducted for TNF $\alpha$ and IL- $1 \beta$ due to the low number of data-points available for these cytokines in the HR and LR cohorts.

Birth weight differences between high and low responders. Bodyweight of pigs, specifically birth weight, has been used as an indicator of growth and survival ${ }^{33}$ and has been previously associated with vaccine responsiveness $^{34}$. The design of this study deliberately excluded the naturally-occurring extremes of piglet birth weight by including only the six piglets per litter with average-litter birth weight. This reduction in birth weight variation may have limited the analysis of birth weight as a biomarker for predicting vaccine responsiveness.

Within the sample population, the range of birth weights, weaning weights, and end weights of the pigs was $1.0-1.9 \mathrm{~kg}, 4.6-10.0 \mathrm{~kg}$, and $19.0-37.7 \mathrm{~kg}$, respectively. LR pigs had lower birth $(\mathrm{p}=0.001)$ and weaning $(\mathrm{p}=0.0018)$ weights compared to HR pigs (Fig. 4a). At the end of the experiment (Day 63), a difference $(\mathrm{p}=0.28)$ in weight between HR and LR pigs was not observed, which may suggest that LR pigs did not have inherent longterm growth impairments (Fig. 4a). Within the HR and LR cohorts, there was a positive correlation $\left(r^{2}=0.68\right.$, $\mathrm{p}<0.005$ ) between birth weight and serum M. hyopneumoniae-specific IgG titer (Fig. 4b). While a correlation between birth weight and serum $M$. hyopneumoniae-specific IgG titer was not evident within the entire sample population $\left(\mathrm{r}^{2}=0.04, \mathrm{p}<0.05\right)$ (Fig. 4c), pigs below the median birth weight $(1.0-1.4 \mathrm{~kg})$ had a lower $(\mathrm{p}=0.046)$ serum M. hyopneumoniae-specific IgG titer than pigs above the median birth weight (1.5-1.9 kg) (Fig. $4 \mathrm{~d})$. Similarly, pigs with a weaning weight below the median weight $(4.6-6.8 \mathrm{~kg})$ had a lower $(\mathrm{p}=0.0478) \operatorname{serum~} M$. hyopneumoniae-specific IgG titer compared to pigs above the median wean weight (6.9-10 kg) (Fig. $4 \mathrm{~d})$.

Correlation analysis of birth weight, plasma IFNy, and vaccine responsiveness. Following observation of the positive correlation between birth weight and serum $M$. hyopneumoniae-specific IgG titer, and the negative correlation between plasma IFN $\gamma$ concentrations and serum M. hyopneumoniae-specific IgG titer, correlation analysis of HR and LR birth weight and plasma IFN $\gamma$ concentrations were found to be negatively correlated $(\mathrm{r}=-0.67, \mathrm{p}<0.05)$ (Fig. 5). Collectively, pigs exhibiting high and low serum M. hyopneumoniae-specific IgG titer following vaccination show disparity in birth weight and plasma IFN $\gamma$ concentrations prior to vaccination. This data provides evidence that the magnitude of vaccine-induced antibody responses may be predicted by biological parameters measured prior to or at the time of vaccination.

\section{Discussion}

While vaccination is one of the most effective tools for preventing and managing infectious diseases, individuals who fail to develop an immune response following vaccination can remain susceptible to infection. The low responders have the potential to transmit infection to others in the population and reduce the proportion of protected individuals needed for herd immunity. Kinome analysis of PBMCs collected prior to primary vaccination led to the discovery of differentially phosphorylated peptides when comparing HR and LR pigs, demonstrating that vaccine responsiveness is associated with unique host cell signaling prior to vaccination. The kinome analysis results implicated differential pro-inflammatory cytokine signaling pathways as a difference between HR and LR pigs. This finding was further validated by analyzing the plasma cytokine concentrations in HR and LR pigs prior 


\begin{tabular}{|c|c|c|c|c|}
\hline Name & Target site & UniProt ID & FC & p-value \\
\hline TRAF6 & $\mathrm{Tyr}^{353}$ & Q9Y4K3 & -2.39 & $4.53 \mathrm{E}-06$ \\
\hline Calmodulin & Tyr ${ }^{99}$ & P62158 & -1.73 & $3.44 \mathrm{E}-05$ \\
\hline gp130 & $\mathrm{Ser}^{782}$ & P40189 & 1.65 & $2.41 \mathrm{E}-04$ \\
\hline BLNK & Tyr $^{178}$ & Q8WV28 & 1.54 & $2.92 \mathrm{E}-04$ \\
\hline NFAT2 & Ser ${ }^{172}$ & O95644 & 1.71 & $3.26 \mathrm{E}-04$ \\
\hline Aktl & $\mathrm{Thr}^{308}$ & P31749 & -2.06 & $4.26 \mathrm{E}-04$ \\
\hline Pyk2 & $\mathrm{Ser}^{399}$ & Q14289 & 1.99 & $4.72 \mathrm{E}-04$ \\
\hline Mek1 & $\operatorname{Ser}^{217}$ & Q02750 & 1.68 & $5.27 \mathrm{E}-04$ \\
\hline Rack1 & Tyr $^{194}$ & P63244 & -2.28 & $1.38 \mathrm{E}-03$ \\
\hline Calmodulin & $\mathrm{Thr}^{79}$ & P62158 & 1.29 & $1.89 \mathrm{E}-03$ \\
\hline MAVS & $\mathrm{Ser}^{233 / 234}$ & Q7Z434 & -1.26 & $1.93 \mathrm{E}-03$ \\
\hline PPP2C $\alpha$ & $\mathrm{Thr}^{307}$ & P67775 & -1.72 & $1.99 \mathrm{E}-03$ \\
\hline Grb10 & $\operatorname{Ser}^{150}$ & Q13322 & 1.58 & $2.43 \mathrm{E}-03$ \\
\hline Aktl & $\mathrm{Thr}^{450}$ & P31749 & 1.37 & $2.71 \mathrm{E}-03$ \\
\hline Syk & $\mathrm{Tyr}^{348}$ & P43405 & 1.40 & $4.15 \mathrm{E}-03$ \\
\hline Kit & Tyr ${ }^{936}$ & P10721 & 1.49 & $4.61 \mathrm{E}-03$ \\
\hline Caspase-8 & Tyr $\mathrm{r}^{448}$ & Q14790 & 1.39 & $5.16 \mathrm{E}-03$ \\
\hline PKAC $\alpha$ & Thr ${ }^{197}$ & P17612 & -2.10 & $5.43 \mathrm{E}-03$ \\
\hline 4E-BP1 & $\mathrm{Thr}^{46}$ & Q13541 & 1.31 & $5.95 \mathrm{E}-03$ \\
\hline TNIK & $\mathrm{Thr}^{181}$ & Q9UKE5 & 2.10 & $8.59 \mathrm{E}-03$ \\
\hline p70S6K & $\mathrm{Ser}^{447}$ & P23443 & -1.56 & $8.69 \mathrm{E}-03$ \\
\hline Kit & $\mathrm{Tyr}^{568 / 570}$ & P10721 & 1.52 & $8.85 \mathrm{E}-03$ \\
\hline IKK- $\alpha$ & $\mathrm{Ser}^{473}$ & O15111 & -1.46 & $9.16 \mathrm{E}-03$ \\
\hline p53 & $\operatorname{Ser}^{15}$ & P04637 & -1.36 & $9.61 \mathrm{E}-03$ \\
\hline Smad6 & $\operatorname{Ser}^{435}$ & O43541 & -2.64 & $1.18 \mathrm{E}-02$ \\
\hline PKCe & $\mathrm{Thr}^{566}$ & Q02156 & -1.24 & $1.33 \mathrm{E}-02$ \\
\hline Kit & $\mathrm{Tyr}^{721}$ & P10721 & 1.26 & $1.40 \mathrm{E}-02$ \\
\hline IRAK1 & Thr ${ }^{100}$ & P51617 & -1.44 & $1.42 \mathrm{E}-02$ \\
\hline NFAT2 & $\operatorname{Ser}^{245}$ & O95644 & -1.56 & $1.60 \mathrm{E}-02$ \\
\hline Cofilin 1 & $\mathrm{Ser}^{2}$ & P23528 & 1.51 & $1.66 \mathrm{E}-02$ \\
\hline HSP60 & $\mathrm{Ser}^{70}$ & P10809 & -1.35 & $1.67 \mathrm{E}-02$ \\
\hline PDGFR $\beta$ & $\mathrm{Tyr}^{740}$ & P09619 & 1.48 & $1.80 \mathrm{E}-02$ \\
\hline Smad3 & $\mathrm{Thr}^{179}$ & P84022 & 1.30 & $1.81 \mathrm{E}-02$ \\
\hline Lyn & $\mathrm{Tyr}^{396}$ & P07948 & 1.47 & $1.82 \mathrm{E}-02$ \\
\hline TBK1 & $\operatorname{Ser}^{172}$ & Q9UHD2 & -1.40 & $1.85 \mathrm{E}-02$ \\
\hline JAK1 & Tyr 220 & P23458 & -2.91 & $1.95 \mathrm{E}-02$ \\
\hline STAT6 & $\mathrm{Tyr}^{641}$ & P42226 & -1.78 & $1.97 \mathrm{E}-02$ \\
\hline HSP27 & $\mathrm{Ser}^{78}$ & P04792 & 1.92 & $2.07 \mathrm{E}-02$ \\
\hline p300 & $\operatorname{Ser}^{2279}$ & Q09472 & -1.27 & $2.12 \mathrm{E}-02$ \\
\hline Cdc42 & Tyr $^{32}$ & P60953 & -1.48 & $2.28 \mathrm{E}-02$ \\
\hline IKK- $\beta$ & Tyr ${ }^{188}$ & O14920 & 1.30 & $2.63 \mathrm{E}-02$ \\
\hline Smad1 & $\operatorname{Ser}^{214}$ & Q15797 & 1.41 & $2.96 \mathrm{E}-02$ \\
\hline MSK2 & $\mathrm{Ser}^{360}$ & O75676 & -1.43 & $3.24 \mathrm{E}-02$ \\
\hline Bcl-2 & $\mathrm{Ser}^{87}$ & P10415 & -1.27 & $3.66 \mathrm{E}-02$ \\
\hline K8 & Tyr $^{267}$ & P05787 & -2.01 & $3.68 \mathrm{E}-02$ \\
\hline CDK2 & Thr ${ }^{160}$ & P24941 & -1.38 & $3.83 \mathrm{E}-02$ \\
\hline fyn & $\mathrm{Tyr}^{531}$ & P06241 & 1.33 & $4.03 \mathrm{E}-02$ \\
\hline CREB & $\operatorname{Ser}^{117}$ & P16220 & 1.27 & $4.11 \mathrm{E}-02$ \\
\hline IKK- $\beta$ & Tyr ${ }^{199}$ & O14920 & -1.30 & $4.52 \mathrm{E}-02$ \\
\hline ERK3 & $\operatorname{Ser}^{189}$ & Q16659 & -1.86 & $4.66 \mathrm{E}-02$ \\
\hline IKK- $\gamma$ & $\mathrm{Ser}^{43}$ & Q9Y6K9 & -1.33 & $4.73 \mathrm{E}-02$ \\
\hline P27kip1 & $\mathrm{Tyr}^{74}$ & P46527 & -1.55 & $4.90 \mathrm{E}-02$ \\
\hline Sek1 & $\mathrm{Ser}^{80}$ & P45985 & -1.28 & $4.94 \mathrm{E}-02$ \\
\hline
\end{tabular}

Table 1. Differentially phosphorylated peptides of PBMCs collected prior to vaccination between HR and LR pigs. Fold-change (FC) in HR relative to LR. 


\begin{tabular}{|c|c|c|c|c|}
\hline Pathway name & Source name & Pathway p-value (corrected) & Number of uploaded genes for this entity & $\begin{array}{l}\text { Number of genes in InnateDB for this } \\
\text { entity }\end{array}$ \\
\hline Innate Immune System & REACTOME & $4.11 \mathrm{E}-20$ & 27 & 563 \\
\hline Signaling by Interleukins & REACTOME & $8.77 \mathrm{E}-17$ & 15 & 110 \\
\hline RANKL & NETPATH & $1.22 \mathrm{E}-16$ & 14 & 84 \\
\hline Immune System & REACTOME & $2.42 \mathrm{E}-16$ & 30 & 1,127 \\
\hline BCR & NETPATH & $3.45 \mathrm{E}-16$ & 16 & 157 \\
\hline Fc epsilon receptor (FCERI) signaling & REACTOME & $1.40 \mathrm{E}-15$ & 16 & 173 \\
\hline JAK-STAT pathway and regulation & $\mathrm{INOH}$ & $2.04 \mathrm{E}-15$ & 18 & 273 \\
\hline TNFalpha & NETPATH & $2.05 \mathrm{E}-15$ & 18 & 270 \\
\hline VEGF signaling pathway & $\mathrm{INOH}$ & $2.08 \mathrm{E}-15$ & 16 & 183 \\
\hline EPO signaling pathway & $\mathrm{INOH}$ & $2.18 \mathrm{E}-15$ & 16 & 181 \\
\hline IL-7 signaling & $\mathrm{INOH}$ & $2.28 \mathrm{E}-15$ & 16 & 180 \\
\hline Leptin & NETPATH & $7.64 \mathrm{E}-15$ & 13 & 96 \\
\hline IL2 & NETPATH & $3.85 \mathrm{E}-14$ & 12 & 81 \\
\hline IL3 & NETPATH & $5.66 \mathrm{E}-14$ & 12 & 84 \\
\hline IL6 & NETPATH & $6.13 \mathrm{E}-14$ & 12 & 85 \\
\hline Prostate cancer & KEGG & $1.18 \mathrm{E}-13$ & 12 & 90 \\
\hline EGFR1 & NETPATH & $1.33 \mathrm{E}-12$ & 19 & 472 \\
\hline BCR signaling pathway & PID NCI & $4.33 \mathrm{E}-12$ & 10 & 63 \\
\hline MyD88-independent cascade & REACTOME & $8.80 \mathrm{E}-12$ & 11 & 97 \\
\hline TRIF-mediated TLR3/TLR4 signaling & REACTOME & $8.80 \mathrm{E}-12$ & 11 & 97 \\
\hline Toll Like Receptor 3 (TLR3) Cascade & REACTOME & $8.80 \mathrm{E}-12$ & 11 & 97 \\
\hline Osteoclast differentiation & KEGG & $9.25 \mathrm{E}-12$ & 12 & 133 \\
\hline Pathways in cancer & KEGG & $9.59 \mathrm{E}-12$ & 16 & 329 \\
\hline Cytokine Signaling in Immune system & REACTOME & $9.60 \mathrm{E}-12$ & 15 & 267 \\
\hline Signaling by NGF & REACTOME & $9.86 \mathrm{E}-12$ & 15 & 271 \\
\hline
\end{tabular}

Table 2. Pathway overrepresentation analysis of differentially phosphorylated peptides in PBMCs collected from HR and LR pigs prior to vaccination. The top 25 pathways are presented.
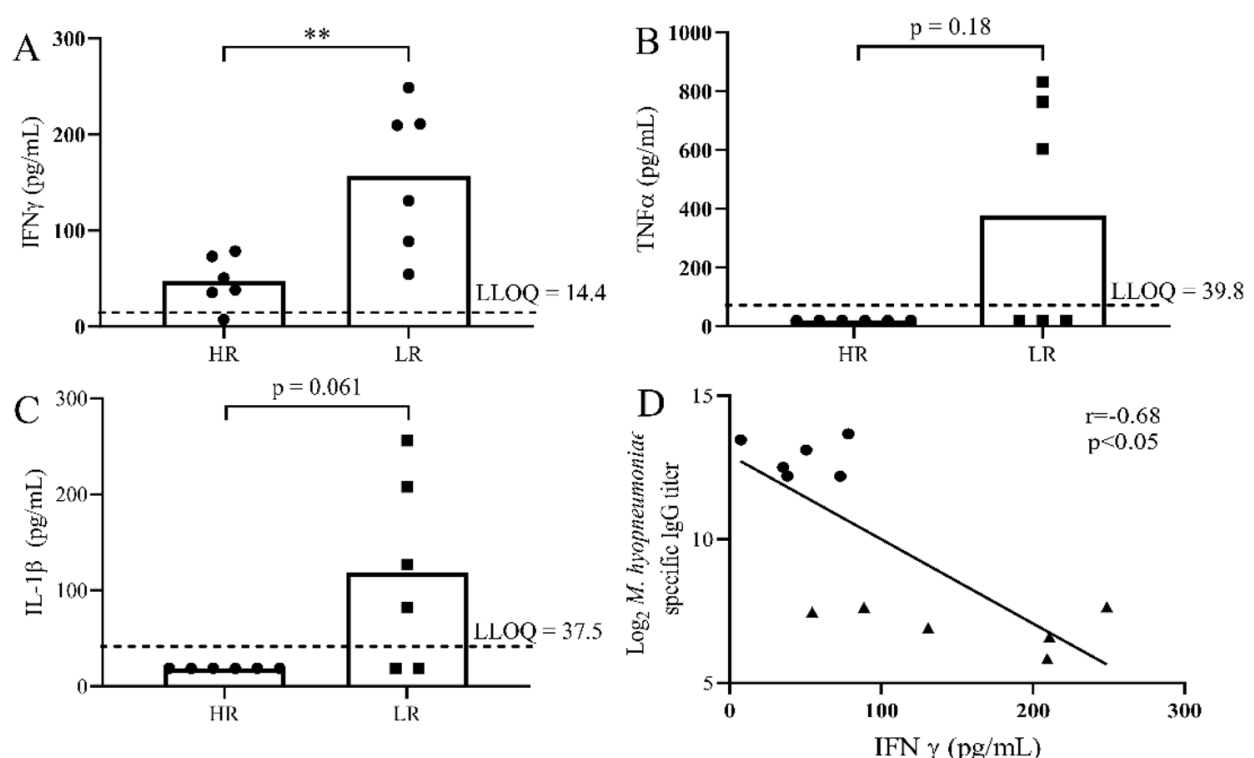

Figure 3. Elevated pro-inflammatory cytokines in low responder plasma compared to high responders prior to vaccination. Concentrations of (A) IFN $\gamma,(\mathbf{B}) \mathrm{TNF} \alpha$, and (C) IL-1 $\beta$ within the plasma of high (HR) and low (LR) responder pigs collected prior to vaccination. (D) Correlation analysis of plasma IFN $\gamma$ concentrations prior to vaccination and post-vaccination serum M. hyopneumoniae-specific IgG titer for HR (circles) and LR (triangles). Bars represent the mean. $n=6$ for both HR and LR groups. Samples below the limit of lower quantification (LLOQ) were included as $1 / 2$ the LLOQ for each assay. ${ }^{* *} \mathrm{p}<0.01$, Mann-Whitney U-test. 

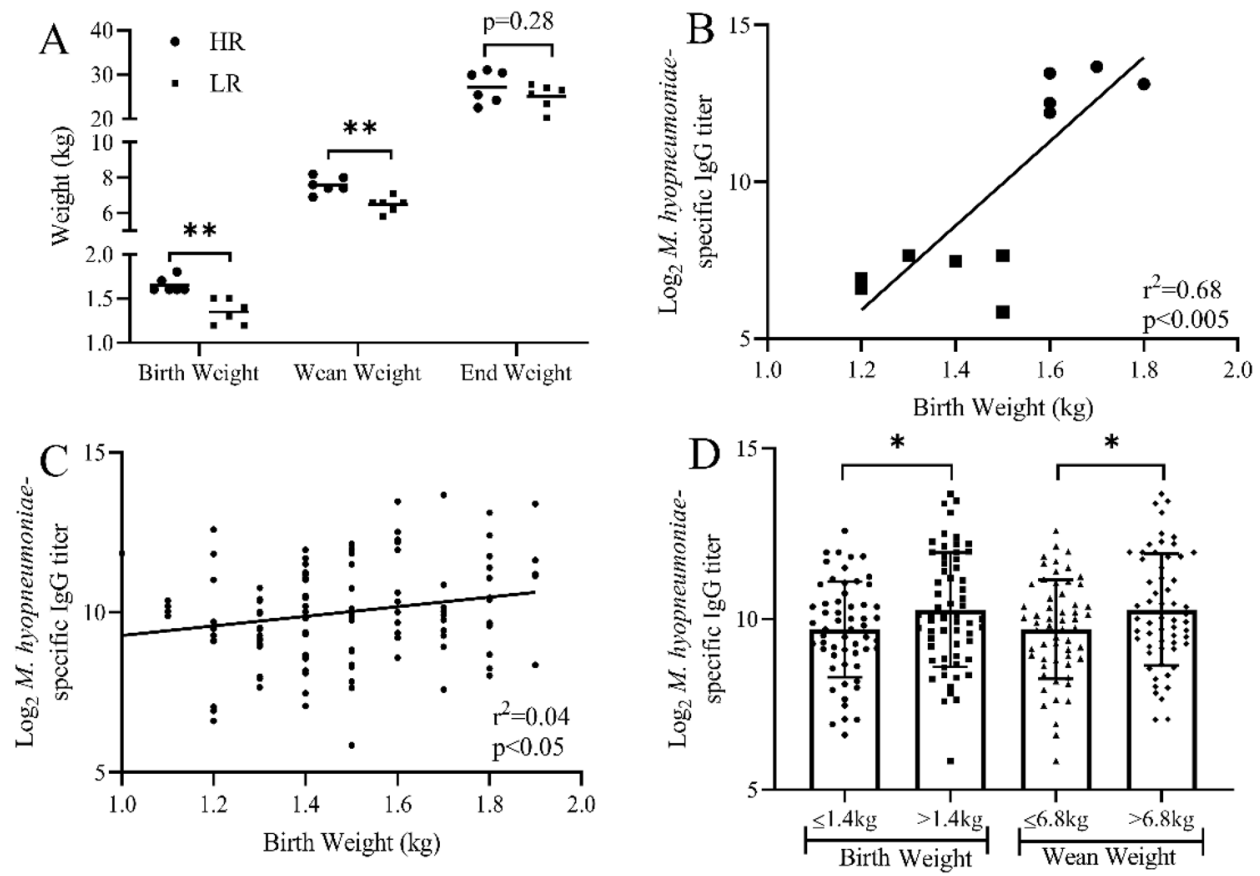

Figure 4. Vaccine-induced antibody responses correlate with both birth and weaning bodyweights of high and low responder pigs. (A) Bodyweight of high (HR; circles) and low (LR; squares) responder pigs at birth, weaning, and at the end of experiment. (B) Correlation analysis of birth weight and serum M. hyopneumoniaespecific IgG titer for HR (circles) and LR (squares) pigs. (C) Correlation analysis of birth weight and serum $M$. hyopneumoniae-specific IgG titer for the sample cohort. (D) Serum M. hyopneumoniae-specific IgG titer of pigs with median birth weight less than (circles) or greater than (squares) $1.4 \mathrm{~kg}$, and pigs withs median wean weight less than (triangles) or greater than (diamonds) $6.8 \mathrm{~kg}$. Data represents mean in (A) and mean \pm SD in (D). Line represents best-fit line of linear regression in (B) and (D). $\mathrm{n}=6$ for both HR and LR groups in (A) and (B), $\mathrm{n}=117$ in $(\mathbf{C})$, and $\mathrm{n} \geq 57$ for each group in (D). ${ }^{*} \mathrm{p}<0.05,{ }^{* *} \mathrm{p}<0.01$, unpaired two-tailed Student's t-test.

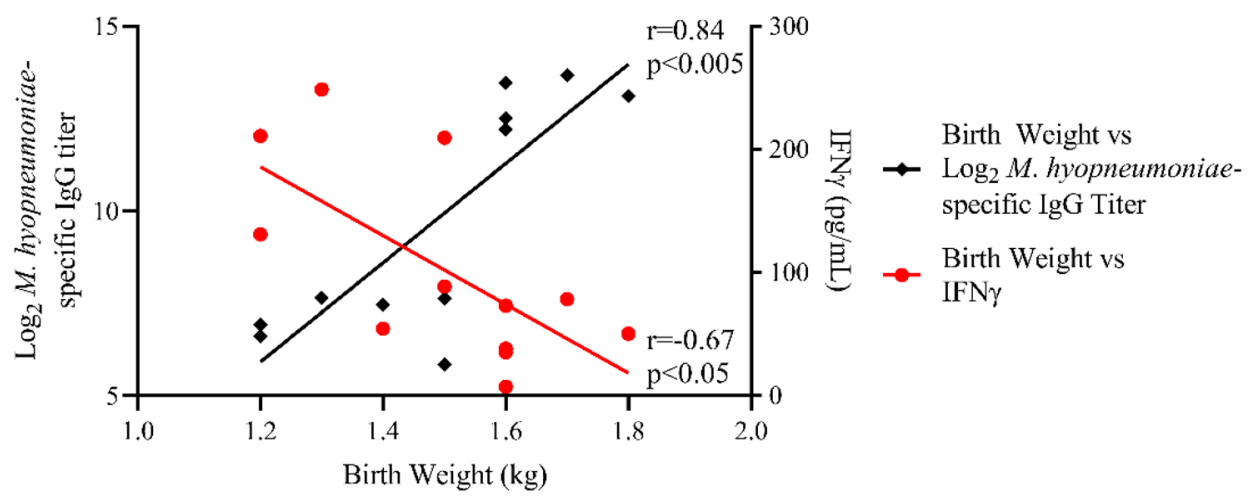

Figure 5. Birth weight of high and low responder pigs correlate with both vaccine responsiveness and plasma IFN $\gamma$ prior to vaccination. Correlation analysis of birth weight and both serum M. hyopneumoniae-specific IgG titer (black) and plasma IFN $\gamma$ concentrations (red) prior to vaccination in high (HR) and (LR) responders. $n=6$ for both HR and LR groups. A single data-point represents a single animal for each analysis. Samples below the limit of lower quantification (LLOQ) were plotted as $1 \frac{1}{2}$ the LLOQ.

to primary vaccination. The analysis revealed differences in circulating plasma cytokine levels that significantly correlated with both animal birth weight and serum specific-IgG titer following booster vaccination. Biomarkers capable of predicting high and low responders prior to vaccination, such as body weight or plasma IFN $\gamma$ concentrations, may facilitate the identification of low responders and eliminate the need to rely on post-vaccination analysis of antibody titers. The positive correlation between birth weight and vaccine-induced antibody titer within high and low responders may present a simple, immediate biomarker for potentially identifying lowresponders at birth, where animals who exhibit low birth weight could potentially be at risk of having a reduced chance of developing a vaccine-induced immune response. Correlations between vaccine responsiveness and 
both piglet birth weight and plasma cytokine concentrations of high and low responders reveal differences that exist prior to vaccination that may have had long-term impacts on vaccine responses, even after two vaccinations.

Several studies have searched for biomarkers and investigated possible mechanisms of vaccine responsiveness by analyzing genetic ${ }^{12,16}$ and dynamic factors such as age ${ }^{35,36}$, weight ${ }^{20}$, and health status ${ }^{21,24}$. Other studies have provided insight into the molecular mechanisms influencing immune responses to vaccination through a variety of -omic approaches $5,7,8,26,32,37$. The current investigation identified biological differences in kinasemediated signaling that were present in PBMCs collected prior to vaccination that discriminated high and low responders. Reduction analysis of the peptide phosphorylation patterns within the PBMCs clustered pigs on the basis of serum specific-IgG titers 11 days following booster vaccination which may suggest the cohorts had a predisposition towards a specific vaccine-induced antibody response. In contrast, transcriptional analysis of PBMCs collected from the same HR and LR pigs used in the current study was only able to discriminate these cohorts at two and six days following primary vaccination ${ }^{32}$. This may reflect the greater sensitivity of kinome analysis to identify cell signaling events in PBMCs at a post-transcriptional level that can directly influence vaccine responsiveness.

In addition to identifying to potential biomarkers of vaccine responsiveness, the kinome analysis generates hypotheses regarding the biological processes that may influence vaccine responses. Within the PBMCs collected prior to vaccination, differences in phosphorylation events between HR and LR pigs revealed altered pro-inflammatory signaling, a finding consistent with the higher $(\mathrm{p}=0.0087)$ plasma IFN $\gamma$ concentration in LR pigs compared to HR pigs. This finding is in agreement with other evidence that pro-inflammatory events prior to vaccination are negatively associated with vaccine responsiveness. In an African population of human subjects vaccinated with the yellow fever 17D vaccine, the cohort with low YF-17D specific-neutralizing antibodies following vaccination displayed greater frequencies of pro-inflammatory monocytes and exhausted natural killer cells prior to vaccination ${ }^{38}$. Fourati et al. reported that seroconversion following hepatitis B virus vaccination of humans was negatively correlated with baseline pro-inflammatory signaling pathways, activated innate immune cells, and upregulation of inflammatory cytokines ${ }^{39}$. The pro-inflammatory cytokine TNFa has also been proposed as a biomarker of vaccine responsiveness in both humans and mice as increased serum TNF $\alpha$ negatively correlated with serum antibody responses and in vitro B-cell responses to stimulation with CpG oligodeoxynucleotides ${ }^{40,41}$. Consistent with these results, LR pigs showed a tendency towards greater levels of TNF $\alpha(p=0.18)$ and IL-1 $\beta(p=0.061)$ compared to HR pigs. The detection of elevated IFN $\gamma$, TNF $\alpha$, and IL- $1 \beta$ in plasma of LR pigs supports the conclusion that circulating pro-inflammatory cytokines prior to vaccination are associated with reduced vaccine-induced antibody responses in pigs to RespiSure-One. However, the current study uses total serum IgG titers following two vaccinations as the only measure of vaccine responsiveness. Additional studies are required to determine if antibody isotypes were also qualitatively different and if T-cell responses were similarly reduced following vaccination.

Further studies are required to determine if IFN $\gamma$ has a direct impact on vaccine responsiveness. IFN $\gamma$ is involved in the upregulation of major histocompatibility complex class I and II, activation of macrophages, and production of pro-inflammatory cytokines. IFN $\gamma$ is a crucial cytokine required for differentiating naïve CD4 T cells into Th1 effector cells to mediate cellular immunity in response to antigens ${ }^{42}$. However, excessive concentrations of IFN $\gamma$ have been found in humans and mice with various autoimmune diseases ${ }^{43-45}$, suggesting IFN $\gamma$ concentrations must be closely controlled for optimal immune responses ${ }^{42}$. As hyper-responsive innate immune systems have been proposed to negatively affect adaptive immune-responses ${ }^{46}$, disproportionate production of pro-inflammatory cytokines may be detrimental to the host and it should be investigated whether elevated plasma IFN $\gamma$ in low responders persists throughout the post-vaccination period. Pro-inflammatory responses have been linked to dysbiosis of the gut microbiome due to antibiotic perturbation, in which broad-spectrum antibiotic use was found to negatively affect H1N1-specific IgG1 and IgA responses following trivalent inactivated influenza vaccination in humans ${ }^{47}$. The use of anti-inflammatory drugs, such as metformin, has improved influenza vaccine-specific antibody responses ${ }^{48}$, and it is suspected that other molecules that reduce systemic inflammation may improve vaccine-induced antibody responses in humans ${ }^{49}$. Collectively, there is strong evidence that pro-inflammatory cytokines can impact cellular responses prior to vaccination which subsequently has a negative effect on vaccine responsiveness.

Low birth weight has been a biomarker for predicting overall health, as within litters of pigs, low birth weight is associated with a greater risk of the piglets being stillbirth $s^{50}$ and greater pre-weaning mortality ${ }^{51}$. This investigation identified an association between body weight and vaccine responsiveness, where LR pigs had lower $(\mathrm{p}<0.005)$ weights at birth and weaning than HR pigs. This association was identified in spite of an experimental design that minimized variance in body weight by selecting pigs of average-litter birth weight. Therefore, the current results may underestimate the strength of the correlation between body weight at both birth and weaning with vaccine responsiveness. A correlation between serum M. hyopneumoniae-specific IgG titer and birth weight was not apparent within the entire study cohort, yet the correlation exists when focusing on the extremes of vaccine responsiveness. A positive relationship between antibody responses and birth weight is consistent with a growing body of evidence which suggests early immune development is crucial in determining the capacity of individuals to generate serum antibody responses to vaccines. Independent studies of humans vaccinated with hepatitis B virus or typhoid fever vaccines have associated low birth weight and reduced serum antibody levels for both vaccines ${ }^{18,52}$. After identifying a correlation between reduced anti-typhoid IgG following typhoid vaccination and low birth weight in a subpopulation of adolescents ${ }^{53}$, elevated pro-inflammatory marker, C-reactive protein, was observed in those individuals with low anti-typhoid IgG responses ${ }^{54}$. The current study also reveals an association between lower birth weights, elevated concentrations of pro-inflammatory cytokines in plasma, and decreased vaccine-induced antibody responses.

In the context of livestock herds, a biomarker, such as plasma cytokine concentrations, could aid in rapidly identifying low responders prior to vaccination. Phenotypic indicators such as birth weight could also be used 
to designate animals who are potentially at greater risk for infection. Tests capable of predicting vaccine responsiveness have the potential to influence herd management decisions and possibly provide biomarkers to improve animal breeding programs. While these findings on piglet birth weight and plasma cytokine expression need to be replicated in an independent herd and trial, the current study provides evidence that two concurrent parameters are associated with vaccine responsiveness in young pigs.

\section{Materials and methods}

Animal care and vaccination. The experimental protocol was approved by the University of Alberta Animal Care and Use Committee-Livestock (AUP00001125) and sample collection was in accordance with the Canadian Council on Animal Care guidelines. The animals used for this study have been described previously ${ }^{32}$. Twenty sows bore litters in which six pigs (three males and three females) with average-litter birth weight were selected. Pigs born from the sows that were not of average-litter birth weight were not recorded in this study, ultimately resulting in a cohort of 117 healthy M. hyopneumoniae-free pigs [(Large White $\times$ Landrace $) \times$ Duroc; 59 male, 58 female]. Three pigs died before the end of the study and were excluded from analyses. Pigs were weaned and weighed at $21 \pm 2$ days of age and subsequently grouped with littermates in nursery pens $(\mathrm{n}=29-30$ pigs/pen). No difference (Ordinary one-way ANOVA, p >0.9999) in M. hyopneumoniae-specific IgG titer was found between pens. Pigs were monitored for changes in weight gain, behaviour, and physical injury throughout the experiment. Pigs were vaccinated intramuscularly with one dose $(1 \mathrm{~mL})$ of RespiSure-One (Zoetis, USA) at 28 days of age and given a booster vaccine at 52 days of age. The trial was terminated when pigs were 63 days of age. In order to address if M. hyopneumoniae was circulating in the herd, a nasal swab from each animal was tested on D0 prior to vaccination to which all animals tested negative for the bacterium. While the maternal serological status was not measured for this study, it was confirmed that at the time of this study and for several years prior to this study, animals within the facility had consistently tested as being M. hyopneumoniae-free. Additionally, the sows were not vaccinated with RespiSure-One to eliminate the concern that vaccine-induced maternal antibodies were transferred to the pigs used in this study.

Serum and plasma collection, and PBMC isolation. Whole blood was collected from the jugular vein

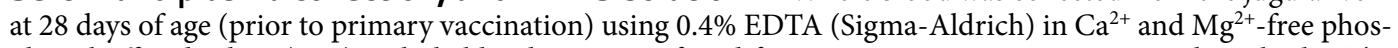
phate-buffered saline (PBS). Whole blood was centrifuged for $20 \mathrm{~min}$ at $1,400 \times \mathrm{g}$ at $20^{\circ} \mathrm{C}$ without brake. The buffy coat layer at the interface of the red blood cells and plasma was collected, diluted 1:2 with room temperature PBS $+0.1 \%$ EDTA, and layered onto $15 \mathrm{~mL}$ of isotonic Ficoll (GE Healthcare) and centrifuged for $20 \mathrm{~min}$ at $2,000 \times \mathrm{g}$ at $20^{\circ} \mathrm{C}$ without brake. PBMCs at the interface of the plasma and isotonic Ficoll were collected and washed twice with ice-cold PBS $+0.1 \%$ EDTA and pelleted by centrifuging for $8 \mathrm{~min}$ at $300 \times \mathrm{g}, 4^{\circ} \mathrm{C}$ with brake. Cells were washed a third time in PBS and pelleted by centrifugation for $8 \mathrm{~min}$ at $150 \times \mathrm{g}, 4^{\circ} \mathrm{C}$ with brake. Viable cell counts were determined by trypan blue (Gibco) exclusion using a hemocytometer. PBMC pellets containing $10 \times 10^{6}$ cells were flash-frozen in liquid nitrogen and stored at $-80^{\circ} \mathrm{C}$.

Whole blood was collected from the jugular vein into anticoagulant-free and EDTA-coated Vacutainer tubes (Becton Dickinson) for the collection of serum and plasma, respectively, at 28 days of age (prior to primary vaccination) and at 63 days of age (11 days after booster vaccination). Serum collection tubes were incubated for $30 \mathrm{~min}$ at room temperature. Serum and plasma tubes were centrifuged for $10 \mathrm{~min}$ at $15,000 \times \mathrm{g}, 4^{\circ} \mathrm{C}$. Samples were aliquoted and stored at $-80^{\circ} \mathrm{C}$.

IgG ELISAs. Methods for determining IgG titer have been described previously ${ }^{32}$. Briefly, $1 \mathrm{~mL}$ of serum from each pig at 63 days of age (11 days after booster vaccination) were shipped to Biovet (Saint-Hyacinthe, QC, Canada) where serum M. hyopneumoniae-specific IgG titers were quantified using an IDEXX ELISA kit (Idexx Laboratories, Inc.). All M. hyopneumoniae-specific IgG titers were transformed using a z-score $\log _{2}$ scale.

Selection of high and low responders. Pigs were stratified by M. hyopneumoniae-specific IgG titer and the six pigs clustering for which there was sufficient sample to perform both kinome profiling and cytokine analysis, were selected to represent the high and low vaccine responders. Several animals were excluded from LR and HR cohorts based on the availability of limited archived samples. These pigs were, however, included in all population analyses for both the serum M. hyopneumoniae-specific IgG titer stratification and the correlation analysis of birth weight and M. hyopneumoniae-specific IgG titer.

Kinome analysis. The design, construction and application of the peptide arrays were performed using previously described protocols ${ }^{55}$. The arrays were fabricated by a commercial provider (JPT Innovative Peptide Solutions) and designed to include peptides representing phosphorylation events associated with a wide variety of signaling pathways. Each array includes nine technical replicates of 282 unique peptides. All kinome experiments were performed on the same day to minimize potential inter-assay variance.

All products were purchased from Sigma-Aldrich unless otherwise stated. Briefly, $10 \times 10^{6} \mathrm{PBMCs}$ were lysed with ice-cold lysis buffer $(20 \mathrm{mM}$ Tris- $\mathrm{HCl}$ [pH 7.5], $150 \mathrm{mM} \mathrm{NaCl}, 1 \mathrm{mM}$ EDTA, $1 \mathrm{mM}$ EGTA, 1\% Triton $\mathrm{X}-100,2.5 \mathrm{mM}$ sodium pyrophosphate, $1 \mathrm{mM} \mathrm{Na}_{3} \mathrm{VO}_{4}, 1 \mathrm{mM} \mathrm{NaF}, 1 \mu \mathrm{g} / \mathrm{mL}$ leupeptin, $1 \mu \mathrm{g} / \mathrm{mL}$ aprotinin, $1 \mathrm{mM}$ PMSF) and incubated for $10 \mathrm{~min}$ on ice. Lysates were centrifuged for $10 \mathrm{~min}$ at $14,000 \times \mathrm{g}, 4^{\circ} \mathrm{C}$. Supernatant was combined with 8:1 activation mix [ $50 \%$ glycerol, $50 \mu \mathrm{M}$ ATP (New England Biolabs), $60 \mathrm{mM} \mathrm{MgCl}, 0.05 \%$ Brij-35, $0.25 \mathrm{mg} / \mathrm{mL}$ bovine serum albumin] for $10 \mathrm{~min}$ on ice. Samples were incubated on the peptide array for $2 \mathrm{~h}$ at $37^{\circ} \mathrm{C}$. Arrays were washed with PBS $+1 \%$ Triton X-100 and submerged in ProQ Diamond phosphoprotein stain (Invitrogen) and incubated for $1 \mathrm{~h}$ with agitation. Arrays were destained with $20 \%$ acetonitrile $+50 \mathrm{mM}$ sodium acetate, $\mathrm{pH} 4.0$ for $10 \mathrm{~min}$. Arrays were washed with distilled deionized water and centrifuged for $5 \mathrm{~min}$ 
at $800 \times \mathrm{g}$ to remove excess moisture. Phosphorylation intensity was collected using a GenePix Professional 4200A Microarray Scanner at 532-560 nm with a $580 \mathrm{~nm}$ filter. Images were captured using the GenePix Pro 6.0 software (MDS) to collect spot intensity ${ }^{30}$.

Peptide-spot intensities were transformed using a variance-stabilizing normalization (VSN) method through the online software, PIIKA (https://saphire.usask.ca/saphire/piika/). Peptides that showed variation in technical replicates via Chi-squared test $\left(\chi^{2}<0.01\right)$ were removed from subsequent analysis. Consistent technical replicates were averaged together, and fold-change (FC) for each peptide was calculated as $F C=2^{d}, d=$ average $_{\text {HRintensity }}-$ average $_{\text {LRintensity. }}$. The $\mathrm{t}$-distributed stochastic neighbour embedding (t-SNE) analysis and hierarchical clustering were conducted using peptides with consistent phosphorylation $\left(\chi^{2}>0.01\right)$. The $\mathrm{t}$-SNE analysis was conducted using the R package Rtsne (https://github.com/jkrijthe/Rtsne) ${ }^{56}$ and visualized using ggplot2 (https://ggplot2.tidyverse.org) ${ }^{57}$. The t-SNE analysis was performed 100 times and the result with the lowest value of the objective function was selected. The construction of the heatmap using PIIKA has been described previously ${ }^{58}$. Hierarchical clustering was conducted using the Pearson correlation distance and McQuitty linkage. Peptides were considered differentially phosphorylated under two given criteria: first, the peptide was consistently phosphorylated according to the Chi-squared test and second, the VSN-transformed phosphorylation intensity of an individual peptide was significantly different (two-tailed Welch's t-test for Unequal Variances, $\mathrm{p}<0.05$ ) between cohorts.

Pathway over-representation analysis. Peptides that were differentially phosphorylated were subjected to pathway over-representation analysis (ORA) using InnateDB ${ }^{59}$, an online software program for querying datasets of genes/proteins against multiple curated databases to determine biologically relevant pathways. ORA was completed using the hypergeometric algorithm with Benjamani-Hochberg correction method, and pathways were considered statistically significant with a false discovery rate (FDR) of $p<0.05$.

Porcine-specific multiplex analysis. Plasma samples had undergone two freeze-thaw cycles prior to multiplex analysis. All incubations were done at room temperature with agitation at $750 \mathrm{rpm}$. Plates were covered in foil to reduce light exposure. All products are from Sigma-Aldrich unless otherwise stated. Following each incubation, plates were washed with PBS pH 7.4 (Gibco) + 0.5\% Tween 20 using a Bio-Plex PRO II wash station (30-s soak, 3 cycles). Standards were diluted 1:4 in New Zealand pig serum to account for serum inhibitory effects while samples were diluted 1:2 and 1:4 in diluent (PBS, $1 \%$ New Zealand pig serum, 0.05\% sodiumazide). Porcine-specific antibodies for interferon $\alpha$ (IFN $\alpha$ ), IFN $\gamma$, interleukin-1 $\beta$ (IL-1 $\beta)$, IL-6, IL-8, IL-12, IL-13, IL-17 $\alpha$ and tumor necrosis factor $\alpha(\mathrm{TNF} \alpha)$ were conjugated to individual BioPlex Max-Plex C magnetic beads (BioRad) following the manufacturer's instructions. TNFa multiplex analysis was conducted on a separate plate to avoid cross-reactivity.

Diluted porcine plasma was incubated for $1 \mathrm{~h}$ with 1,200 beads/well in a Fluortrac $20096 \mathrm{~F}$ microplate (Greiner Bio-One). Following one wash, samples were incubated for $30 \mathrm{~min}$ with biotinylated antibodies specific for the corresponding porcine-cytokine. Following another wash, samples were incubated for 30 min with $5 \mu \mathrm{g} /$ $\mathrm{mL}$ streptavidin R-phycoerythrin conjugate (ThermoFisher Scientific). After a final wash, samples were incubated for $5 \mathrm{~min}$ with TE buffer ( $50 \mathrm{mM}$ Tris, $25 \mathrm{mM}$ EDTA, pH 8.0). Plates were read on a BioPlex 200 reader (BioRad Laboratories Inc.) with the settings " 50 beads per region, 45 -s time-out, and $60 \mu \mathrm{L}$ volume". All replicates and dilution factors for each animal were averaged together for a final concentration. Technical replicates below the lower limit of quantification (LLOQ) were not calculated in the average result. Samples below the limit of detection were recorded as $1 / 2$ the LLOQ value. LLOQ values for all cytokines are given: IFNa $(1.57 \mathrm{pg} / \mathrm{mL}), \mathrm{IFN} \gamma$ (14.4 pg/mL), IL-1 $3(37.5 \mathrm{pg} / \mathrm{mL})$, IL-6 (40.3 pg/mL), IL-8 (2.72 pg/mL), IL-12 (39.1 pg/mL), IL-13 (40.0 pg/ $\mathrm{mL})$, IL-17a $(15.5 \mathrm{pg} / \mathrm{mL})$, and TNFa $(39.8 \mathrm{pg} / \mathrm{mL})$.

Data and statistical analysis. All data analysis and data visualization were performed using GraphPad Prism version 8.1 (GraphPad Software, San Diego, California USA, https://www.graphpad.com). The following statistical tests were conducted on all peptides that showed consistent phosphorylation among technical replicates (Chi-squared test, $\chi^{2}>0.05$ ). The $\log _{2}$-transformed serum $M$. hyopneumoniae-specific IgG titer data and the VSN-transformed kinome data was determined to be normally distributed (Kolmogorov-Smirnov test, $\mathrm{p}>0.1$ ). The birth weight of piglets is assumed to follow a normal distribution in nature. The sample size under consideration $(\mathrm{n}=117)$ is large, samples were measured independently, and the mean $(1.48 \mathrm{~kg})$ is approximately the median $(1.5 \mathrm{~kg})$. Plasma cytokine concentrations were not determined to be normally distributed (Kolmogorov-Smirnov test, $\mathrm{p}<0.1$ ). A two-tailed unpaired Student's t-test was conducted to analyze differences in serum M. hyopneumoniae-specific IgG titer, birth weight, weaning weight, or end weight between HR and LR cohorts. A two-tailed Welch's T-test for Unequal Variances was conducted to determine differences of means between HR and LR phosphorylation intensities as the variances of all peptides did not show homogeneity (F-test of equality of variances, $\mathrm{p}<0.05$ ). A two-tailed Mann-Whitney U-test was conducted to analyze differences in plasma cytokine concentrations between HR and LR cohorts. A Pearson linear regression was conducted for the correlation analysis of serum M. hyopneumoniae-specific IgG titer and birth weight. A Spearman Rank Correlation was conducted for the correlation analysis of serum $M$. hyopneumoniae-specific IgG titer and plasma IFN $\gamma$ concentrations, and birth weight and plasma IFN $\gamma$ concentrations. P-values were considered statistically significant at $\mathrm{p}<0.05$.

Received: 3 December 2019; Accepted: 17 May 2020

Published online: 14 July 2020 


\section{References}

1. Thornton, P. K. Livestock production: recent trends, future prospects. Philos. Trans. R. Soc. B Biol. Sci. 365, 2853-2867 (2010).

2. Laxminarayan, R. et al. Antibiotic resistance-The need for global solutions. Lancet Infect. Dis. 13, 1057-1098 (2013).

3. Heininger, U. et al. The concept of vaccination failure. Vaccine 30, 1265-1268 (2012).

4. Wang, I.-M., Bett, A. J., Cristescu, R., Loboda, A. \& ter Meulen, J. Transcriptional profiling of vaccine-induced immune responses in humans and non-human primates. Microb. Biotechnol. 5, 177-187 (2012).

5. Nakaya, H. I. et al. Systems biology of vaccination for seasonal influenza in humans. Nat. Immunol. 12, $786-795$ (2011).

6. Rechtien, A. et al. Systems vaccinology identifies an early innate immune signature as a correlate of antibody responses to the ebola vaccine rVSV-ZEBOV. Cell Rep. 20, 2251-2261 (2017).

7. Bartholomeus, E. et al. Transcriptome profiling in blood before and after hepatitis B vaccination shows significant differences in gene expression between responders and non-responders. Vaccine 36, 6282-6289 (2018).

8. Querec, T. D. et al. Systems biology approach predicts immunogenicity of the yellow fever vaccine in humans. Nat. Immunol. 10, $116-125$ (2009).

9. Tsang, J. S. et al. Global analyses of human immune variation reveal baseline predictors of postvaccination responses. Cell 157, 499-513 (2014).

10. Wilkie, B. \& Mallard, B. Selection for high immune response: an alternative approach to animal health maintenance?. Vet. Immunol. Immunopathol. 72, 231-235 (1999).

11. Newport, M. J. et al. Genetic regulation of immune responses to vaccines in early life. Genes Immun. 5, 122-129 (2004).

12. Posteraro, B. et al. The link between genetic variation and variability in vaccine responses: Systematic review and meta-analyses. Vaccine 32, 1661-1669 (2014).

13. Ovsyannikova, I. G. et al. The role of polymorphisms in Toll-like receptors and their associated intracellular signaling genes in measles vaccine immunity. Hum. Genet. 130, 547-561 (2011).

14. Dhiman, N. et al. Variations in measles vaccine-specific humoral immunity by polymorphisms in SLAM and CD46 measles virus receptors. J. Allergy Clin. Immunol. 120, 666-672 (2007).

15. Wang, C. et al. HLA and cytokine gene polymorphisms are independently associated with responses to hepatitis B vaccination. Hepatology 39, 978-988 (2004).

16. Poland, G. A., Ovsyannikova, I. G. \& Jacobson, R. M. Immunogenetics of seasonal influenza vaccine response. Vaccine 26S, D35-40 (2008).

17. Frasca, D. \& Blomberg, B. B. Inflammaging decreases adaptive and innate immune responses in mice and humans. Biogerontology 17, 7-19 (2016).

18. Moore, S. E. et al. Birth weight predicts response to vaccination in adults born in an urban slum in Lahore. Pakistan. Am. J. Clin. Nutr. 80, 453-459 (2004).

19. Park, H.-L. et al. Obesity-induced chronic inflammation is associated with the reduced efficacy of influenza vaccine. Hum. Vaccin. Immunother. 10, 1181-1186 (2014).

20. Sheridan, P. A. et al. Obesity is associated with impaired immune response to influenza vaccination in humans. Int. J. Obes. 36, 1072-1077 (2012).

21. Burns, V. E., Carroll, D., Ring, C. \& Drayson, M. Antibody response to vaccination and psychosocial stress in humans: Relationships and mechanisms. Vaccine 21, 2523-2534 (2003).

22. Eibl, N. et al. Impaired Primary immune response in type-1 diabetes: results from a controlled vaccination study. Clin. Immunol. 103, 249-259 (2002).

23. Crowe, S. R. et al. Influenza vaccination responses in human systemic lupus erythematosus: Impact of clinical and demographic features. Arthritis Rheum. 63, 2396-2406 (2011).

24. Frasca, D., Diaz, A., Romero, M., Landin, A. M. \& Blomberg, B. B. Cytomegalovirus (CMV) seropositivity decreases B cell responses to the influenza vaccine. Vaccine 33, 1433-1439 (2015).

25. Gaucher, D. et al. Yellow fever vaccine induces integrated multilineage and polyfunctional immune responses. J. Exp. Med. 205, 3119-3131 (2008).

26. Islam, M. A. et al. Deciphering transcriptome profiles of peripheral blood mononuclear cells in response to PRRSV vaccination in pigs. BMC Genom. 17, 641 (2016)

27. Arsenault, R. J. et al. Altered toll-like receptor 9 signaling in Mycobacterium avium subsp. paratuberculosis-infected bovine monocytes reveals potential therapeutic targets. Infect. Immun. 81, 226-237 (2013).

28. Robertson, A. J. et al. Identification of developmentally-specific kinotypes and mechanisms of Varroa mite resistance through whole-organism, kinome analysis of honeybee. Front. Genet. 5, 139 (2014).

29. Régnier, M. et al. Identification of signaling pathways targeted by the food contaminant FB1: Transcriptome and kinome analysis of samples from pig liver and intestine. Mol. Nutr. Food Res. 61, 1700433 (2017).

30. Määttänen, P. et al. Divergent immune responses to Mycobacterium avium subsp. paratuberculosis infection correlate with kinome responses at the site of intestinal infection. Infect. Immun. 81, 2861-2872 (2013).

31. Arsenault, R. J. et al. Mycobacterium avium subsp. paratuberculosis inhibits gamma interferon-induced signaling in bovine monocytes: Insights into the cellular mechanisms of Johne's disease. Infect. Immun. 80, 3039-3048 (2012).

32. Munyaka, P. M. et al. Characterization of whole blood transcriptome and early-life fecal microbiota in high and low responder pigs before, and after vaccination for Mycoplasma hyopneumoniae. Vaccine 37, 1743-1755 (2019).

33. Milligan, B. N., Dewey, C. E. \& de Grau, A. F. Neonatal-piglet weight variation and its relation to pre-weaning mortality and weight gain on commercial farms. Prev. Vet. Med. 56, 119-127 (2002).

34. Zimmermann, P. \& Curtis, N. Factors that influence the immune response to vaccination. Clin. Microbiol. Rev. 32, e00084-e118 (2019).

35. Lambert, N. D., Ovsyannikova, I. G., Pankratz, V. S., Jacobson, R. M. \& Poland, G. A. Understanding the immune response to seasonal influenza vaccination in older adults: A systems biology approach. Expert Rev. Vaccines 11, 985-994 (2012).

36. Panda, A. et al. Age-associated decrease in TLR function in primary human dendritic cells predicts influenza vaccine response. J. Immunol. 184, 2518-2527 (2010).

37. Thompson-Crispi, K. A. et al. A genome-wide association study of immune response traits in Canadian Holstein cattle. BMC Genom. 15, 559 (2014).

38. Muyanja, E. et al. Immune activation alters cellular and humoral responses to yellow fever 17D vaccine. J. Clin. Invest. 124, 3147-3158 (2014).

39. Fourati, S. et al. Pre-vaccination inflammation and B-cell signalling predict age-related hyporesponse to hepatitis B vaccination. Nat. Commun. 7, 10369 (2016)

40. Frasca, D. et al. A molecular mechanism for TNF-a-mediated downregulation of B cell responses. J. Immunol. 188, 279-286 (2012).

41. Frasca, D., Diaz, A., Romero, M., Landin, A. M. \& Blomberg, B. B. High TNF-a levels in resting B cells negatively correlate with their response. Exp. Gerontol. 54, 116-122 (2014).

42. Schoenborn, J. R. \& Wilson, C. B. Regulation of interferon- $\gamma$ during innate and adaptive immune responses. Adv. Immunol. 96, 41-101 (2007) 
43. Parronchi, P. et al. Type 1 T-helper cell predominance and interleukin-12 expression in the gut of patients with Crohn's disease. Am. J. Pathol. 150, 823-832 (1997).

44. Kumar, P. et al. Interferon $\gamma$ and glycemic status in diabetes associated with chronic pancreatitis. Pancreatology 12, 65-70 (2012).

45. Harigai, M. et al. Excessive production of IFN- $\gamma$ in patients with systemic lupus erythematosus and its contribution to induction of b lymphocyte stimulator/B cell-activating factor/TNF ligand superfamily-13B. J. Immunol. 181, 2211-2219 (2008).

46. Pellanda, L. C. et al. Low birth weight and markers of inflammation and endothelial activation in adulthood: the ARIC study. Int. J. Cardiol. 134, 371-377 (2009).

47. Hagan, T. et al. Antibiotics-driven gut microbiome perturbation alters immunity to vaccines in humans. Cell 178, 1313-1328.e13 (2019).

48. Diaz, A. et al. Metformin improves in vivo and in vitro B cell function in individuals with obesity and Type-2 diabetes. Vaccine 35, 2694 (2017).

49. Alter, G. \& Sekaly, R. P. Beyond adjuvants: Antagonizing inflammation to enhance vaccine immunity. Vaccine 33(Suppl 2), B55-B59 (2015).

50. Quiniou, N., Dagorn, J. \& Gaudré, D. Variation of piglets' birth weight and consequences on subsequent performance. Livest. Prod. Sci. 78, 63-70 (2002).

51. Milligan, B. N., Fraser, D. \& Kramer, D. L. Within-litter birth weight variation in the domestic pig and its relation to pre-weaning survival, weight gain, and variation in weaning weights. Livest. Prod. Sci. 76, 181-191 (2002).

52. Han, K. et al. Revaccination of non- and low-responders after a standard three dose hepatitis B vaccine schedule. Hum. Vaccin. Immunother. 8, 1845-1849 (2012).

53. McDade, T. W., Beck, M. A., Kuzawa, C. \& Adair, L. S. Prenatal undernutrition, postnatal environments, and antibody response to vaccination in adolescence. Am. J. Clin. Nutr. 74, 543-548 (2001).

54. McDade, T. W., Adair, L., Feranil, A. B. \& Kuzawa, C. Positive antibody response to vaccination in adolescence predicts lower C-reactive protein concentration in young adulthood in the Philippines. Am. J. Hum. Biol. 23, 313-318 (2011).

55. Arsenault, R., Griebel, P. \& Napper, S. Peptide arrays for kinome analysis: New opportunities and remaining challenges. Proteomics 11, 4595-4609 (2011).

56. Krijthe, J. H. Rtsne: T-distributed stochastic neighbor embedding using barnes-hut implementation. R package version 0.15. https:// github.com/jkrijthe/Rtsne (2015).

57. Wickham, H. ggplot2: Elegant Graphics for Data Analysis (Springer-Verlag, New York, 2016).

58. Li, Y. et al. A systematic approach for analysis of peptide array kinome data. Sci. Signal. 5, pl2 (2012).

59. Breuer, K. et al. InnateDB: Systems biology of innate immunity and beyond-recent updates and continuing curation. Nucleic Acids Res. 41, D1228-D1233 (2013).

\title{
Acknowledgements
}

The authors thank Natalie Diether and the University of Alberta Swine Research and Technology Centre staff for their help with the animal trial (animal care, animal monitoring, vaccination and sample collection), and Donna Dent for the training and technical support when using the Bioplex 200. Zoetis are thanked for the gift of the RespiSure-One used in the study. This work was financially supported by the Alberta Agriculture and Forestry project (2014F034R) as well as the Saskatchewan Agriculture Development Fund (20170127). Published as VIDO-InterVac manuscript series no. 885.

\section{Author contributions}

S.N., J.W. and G.P. designed and conceived of the research. J.W. collected the samples and ELISA data. S.L., and E.S. performed the kinome experiments and their analyses. S.L. performed the cytokine analysis. A.K. and C.D. implemented the computer code and supporting algorithms. S.L., J.W. and A.F. conducted the data and statistical analyses. S.L., S.N., P.G., and A.F. prepared the figures. S.L., S.N., P.G., and A.F. wrote the manuscript. G.P., J.W., and S.N. provided the funding for the study.

\section{Competing interests}

The authors declare no competing interests.

\section{Additional information}

Correspondence and requests for materials should be addressed to S.N.

Reprints and permissions information is available at www.nature.com/reprints.

Publisher's note Springer Nature remains neutral with regard to jurisdictional claims in published maps and institutional affiliations.

\begin{abstract}
Open Access This article is licensed under a Creative Commons Attribution 4.0 International cc) License, which permits use, sharing, adaptation, distribution and reproduction in any medium or
format, as long as you give appropriate credit to the original author(s) and the source, provide a link to the Creative Commons license, and indicate if changes were made. The images or other third party material in this article are included in the article's Creative Commons license, unless indicated otherwise in a credit line to the material. If material is not included in the article's Creative Commons license and your intended use is not permitted by statutory regulation or exceeds the permitted use, you will need to obtain permission directly from the copyright holder. To view a copy of this license, visit http://creativecommons.org/licenses/by/4.0/.
\end{abstract}

(C) The Author(s) 2020 